Journal of Energy and
Environmental Sulial publication of the International Society for Energy, Environment and Sustainability (ISEES)
Journal homepage : www.jees.in

\title{
Earth's Sustainable Energy in the Universe - Fusion as in the Sun
}

\author{
JS Rao \\ The Vibration Institute of India, Bangalore, India
}

\section{A R T I C L E I N F O}

Received : 22 January 2018

Revised : 16 March 2018

Accepted : 10 April 2018

Keywords:

Sustainable energy, gravitation waves,

radiation, thermonuclear fusion

\author{
A B S T R A C T
}

There are only two energy sources for earth; gravitational waves as the universe expands with collisions of massive black holes and the energy radiating from Sun with thermonuclear fusion reactions constantly taking place until it dies. To have sustainable energy until earth dies Thermonuclear Fusion reaction should be brought down to the earth similar as in Sun before exhausting Fossil fuels and other derived forms of energy. This paper discusses about various efforts since Einstein's discovery of gravitational waves on some of the fundamental aspects on energy in the Universe and in particular Earth's needs.

\section{Introduction}

The age of the universe is approximately placed at 13.82 billion years. Its total energy content is placed at zero with matter and energy exchanging in their forms. The earth is formed around 4.54 billion years ago from the solar nebula. In the early periods much of the Earth was molten because of frequent collisions with other bodies leading to extreme volcanism. Over time, the earth cooled, causing the formation of a solid crust, and allowing liquid water to exist on the surface.

The Sun is the source of most of the energy that drives the biological and physical processes in the world around us - in oceans and on land it fuels plant growth that forms the base of the food chain, and in the atmosphere it warms air which drives our weather. The earth has seen several ice ages in its life time.

With the close of the last ice age about 13,000 BC more clement weather patterns resulted and humans ventured out of caves and other natural shelters. Man has become nomadic since then and settled to village life. Archimedes times about 2200 years ago produced some useful technologies without the aid of science. Science as we know today is just about four centuries old, Solids, Fluids, Thermodynamics and Electromagnetism are all borne in this period and have remained basic sciences and laws of nature. Newton's law of attraction says that a particle attracts every other particle in the universe using a force that is directly proportional to the product of their masses but also inversely proportional to the square of the distance between them. This is true when the particles have velocities less than that of speed of light. For Einstein just about 100 years ago, Gravity is distortion in the fabric of space and time; Gravity is not a Force of Attraction as Newton said. According to this theory, Matter tells space how to curve; space tells how matter should move. He predicted in 1916 gravitational waves, which are ripples in the curvature of space-time, travelling outward from the source stretching and squeezing the space as they go (similar to ripples in a pond). They transport energy as gravitational radiation. Since Einstein, scientific work in energy field concentrated on two fronts: 1. Particle Research and 2. Gravitational Waves.

The European Organization for Nuclear Research established in 1954, known as CERN (Conseil Européen pour la Recherche Nucléaire), is the first research organization operating the largest particle physics laboratory in the world located near Geneva on the Franco-Swiss border with 22 member states predominantly European. India and Pakistan are associate members and several countries are observers.

CERN's main function is to provide the particle accelerators for highenergy physics research - as a result, numerous experiments have been constructed at CERN through international collaborations. The laboratory was originally devoted to the study of atomic nuclei, but was soon applied to higher-energy physics, concerned mainly with the study of interactions between subatomic particles.

CERN currently operates the Large Hadron Collider (LHC), a worldwide scientific cooperation project. The LHC tunnel $27 \mathrm{~km}$ circumference is located $100 \mathrm{~m}$ underground. CERN's existing PS (Proton Synchrotron)/SPS (Super Proton Synchrotron) accelerator complexes are used to pre-accelerate protons and lead ions which are then injected

\footnotetext{
*Corresponding Author: rao_js@yahoo.com
} 
into the LHC. Several experiments are located along the LHC; each of them studies particle collisions from a different aspect, and with different technologies. Construction for these experiments required an extraordinary engineering effort. The initial particle beams were injected into the LHC August 2008. LHC is designed to accelerate protons to energies of seven trillion electron volts and then smash them together in search of new particles and perhaps new forces of nature, see Overbye (2008).

In July 2012, CERN scientists announced the discovery of a new subatomic particle that was later confirmed to be the Higgs boson, see Cho (2012). In March 2013, CERN announced that the measurements performed on the newly found particle allowed it to conclude that this is a Higgs boson.[28] In early 2013, the LHC was deactivated for a twoyear maintenance period, to strengthen the electrical connections between magnets inside the accelerator and for other upgrades. Subsequently Proton beams successfully circulated in the 27-kilometer ring in both directions and with a record-breaking energy of 6.5 trillion electron volts.

Earth is a macro object, radius of 6.371 million $\mathrm{m}$ on one hand and on the other a pale blue dot in space with gravitational waves zipping across at speed of light. There are only two energy sources for the earth:

1. Energy from Sun with its activity oscillating between high to low causing warming and freezing conditions.

2. A minute amount of energy from passing gravitational waves occurring almost continuously with the expanding Universe containing trillions and trillions of moving objects.

Man having domesticated animals was looking for machines to do his chores faster. Archeological evidence in China indicates surface mining of coal and household usage after approximately $3490 \mathrm{BC}$, see, Chang and Robinson (2017). Hero (10-70 AD) of Alexandria made the first reaction steam turbine but he did not have any clue of what application he can put this device except that of opening temple doors, see Rao (2011). In a similar manner, Vitruvius used Overshot Wheels in development of Hydro Energy, Windmills of $7^{\text {th }}$ century AD ... without the aid of Science of $17^{\text {th }}$ century.

Its de Laval and Edison in 1883-84 developed the first electric power plant and since then man has been looking at energy for his needs in ever increasing scale that is unheard of in human history. The energy for these purposes so far came from Sun in the form of Fossil Fuels, Coal and Oil with external and internal combustion. These energy sources stored from millions of years in the earth and are being depleted rapidly. The derived renewable energies such as wind, ocean and solar panels ... are so small in comparison to man's needs; we have to look for sustainable energies such as matter (atoms) that can be converted to useful energy through Einstein's equation $E=m c^{2}$ of 1903 evolved the basis of Nuclear Fission and Fusion. While both are feasible to be sustainable energies, fission has a distinct disadvantage of creating harmful and dreaded radiation and thus mankind in general is in favor of discontinuing this route though the technology involved is fairly ordinary. It's the fusion process that is attractive as a sustainable energy as it takes place in the Universe in all the stars; however, it involves hitherto unfeasible technologies. Thanks to superconductivity, we can bring Sun to Earth directly, and thus create a sustainable and abundant energy source (as long as earth lives and is safe for humanity).

Regarding the second source of energy to the earth it is just two years ago we are able to demonstrate Einstein's prediction hundred years ago to come realistic by measurement of strain as low as $10^{-21}$ using two American Laser Interferometer Gravitational Wave (Ligo) laboratories in Livingstone, Louisiana and Hamden, Washington and an Italian laboratory, Virgo near Pisa working closely with CERN.

\section{Gravitational Waves}

Though Einstein's theory is well accepted, there was no direct evidence until the detection of gravitational waves on 14 Sept 2015 announced on 11 Feb 2016 by David Reitze - Executive Director of LIGO (Laser Interferometry Gravitational Observatory) in USA. Analysis of the waves according to Einstein's theory suggests they originated from a system of two black holes, each with the mass of about 30 Suns that gravitationally drew closer to each other and the resulting gravitational waves reached the earth 1.3 billion years later, see Reitze (2016).

Besides two Ligos in USA, there is one in Pisa, Italy called Virgo with collaboration of six countries in Europe. Ligo works on principle pf Michelson (1881) Interferometer available in most of the Physics laboratories. However it is designed to measure a distance equal to, as small as, one hundredth of diameter of a Proton in the Nucleus of an Atom the measurement accuracy one hundred billion billionth of a difference in length. Here, two laser beams travel 400 times across the 4 $\mathrm{km}$ length in each of the arms perpendicular to each other between the mirrors unlike a demonstration bench in a laboratory.

The Ecliptic is the circular path on the celestial sphere that the Sun appears to follow over the course of a year. Earth's rotational axis is not perpendicular to its orbital plane and therefore its equatorial plane is not coplanar with the ecliptic plane, but is inclined to it by an angle of about $23.4^{\circ}$. The celestial equator crosses the ecliptic at two points, equinoxes, every year. From Hubble's law (1929) it can be shown that earth as a particle sees the gravitational waves emanating from southern celestial hemisphere and these waves will be normal to the ecliptic at a given time. The gravitational signal of 15 September 2015 first reached Livingston, and then, traveling at the speed of light, reached Hanford seven thousandths of a second later. The measurement has shown that the earth was subjected to $10^{-21}$ amplitude of strain (change in length over original length).

Alfred Wegner, a contemporary of Einstein, in 1912 referred to the ancient supercontinent of the earth as Pangaea (meaning Entire Earth in Greek) and proposed the theory of continental drift. While studying the world map in 1910, he noticed that the coastlines of eastern South America and western Africa fit together, like jigsaw puzzle pieces. He cited four evidences: 1. apparent fit of the continents, 2. Distribution of Fossils across the Continents from Triassic era 250 million years ago, 3. Perfect Match of Same type and Rock and Mountain Correlation across continents and 4. Glacial striation areas in the midst of tropics. The forces behind the continental drift were somewhat assumed by Wegener based on the four evidences that got separated as Pangaea split. Wegener however could not explain what force was driving the motion of the continents over hundreds of millions of years. Despite publishing a large body of compelling fossil and rock evidence for his theory between 1912 and 1929 , his theory was first rejected. It was only in the 1960s that continental drift theory that gained acceptance.

Now we seem to find the reason for the drift, the gravitational waves that came from southern hemisphere during the last 300 million years from the collisions and merger of the black holes probably moved the Pangaea northwards and in the process separated the continents as well. What is the energy coming from gravitational waves? How is it different from the radiation energy coming from the Sun and radiated away into space?

To determine this energy we can use the strain measurement from Ligo on the earth imparted by gravitational waves. While it would be almost an impossible task to do so in the last century, now we can depend on Simulation Based Engineering Science by bringing the Science of $17^{\text {th }}$ century to Engineering directly rather than approximate engineering approach evolved between the two world wars in Stanford University, Palo Alto, USA, see Rao (2018). This is possible by numerical solvers of non-homogenous linear equations of the size of millions of order through modern computers in place of log tables and slide rules. Finite Element method is used in this process. The medium, solid or fluid is divided into large number of finite solid elements such as the earth in the present case and each element is used exactly in accordance to exact sciences and solved on a computer, for example, strain in this case. Or we can impose the measured strain and determine what energy level will cause that strain, see Rao (2016).

First we make the Computer Aided Design (CAD) Model of the Earth. Despite its enormous size this can be accomplished by modern software. The earth has basically three parts; the outer layer with the crust and oceans (overall radius 6.371 million $\mathrm{m}$ ); the Mantle of radius 3.472 million $\mathrm{m}$ and Hot Iron Core of radius of 3.374 million $\mathrm{m}$. Unfortunately there are no codes available to make this large size as a finite element mesh; therefore the earth is modeled down to a scale of one to a million. The mass of the earth is $5.972 \times 10^{24} \mathrm{~kg}$, with mean density $5514 \mathrm{~kg} / \mathrm{m}^{3}$. The black-body temperature is $254.0^{\circ} \mathrm{K}$. The average Poisson's ratio is taken as 0.25 . The top of the mantle is $870^{\circ} \mathrm{C}$; towards the bottom, temperature is about $2,200-3,700^{\circ} \mathrm{C}$. For simplicity the meshed model made had 9944 elements with 9841 nodes. The strain level applied to this model is $10^{-21}$. Since the model is made one million times smaller, a corrected value of strain $10^{-12}$ is applied (the details of derivation are not given here). The Strain Energy integrated over the volume is $1.45 \times 10^{-10}$ J.

This energy is given to earth as a pale blue dot with a gravitational wave passing through the Universe at the speed of light. As the wave passes through it normal to ecliptic at the time of the year imparts energy like a sweep pushing things in its way in about 0.4 seconds. This energy from the waves almost occurring continuously may be responsible for pushing Pangaea over hundreds millions of years and is probably the answer to Wegener's search that resulted in continental drift. As an example the Himalayan mountain range and Tibetan plateau were formed as a result of the collision between the Indian Plate and Eurasian Plate which began 50 million years ago and continues today. 
Orogeny in Geology deals with forces and events that lead to a large structural deformation of the Earth's lithosphere due to the interaction between tectonic plates; a continental plate is crumpled and pushed upwards to form mountain ranges. The asthenosphere is highly viscous and mechanically weak and ductile deforming region of the upper mantle of the Earth. It lies below the lithosphere, at depths between approximately 80 and $200 \mathrm{~km}$ below the earth's surface. Plate tectonics deals with largescale motion of the lithosphere of the earth and is based on Wegner's continental drift theory.

Tectonic plates move because the lithosphere has greater strength than the underlying asthenosphere. Also, the variations in lateral density of the mantle result in convection. Plate movement is attributed to the motion of the seafloor due to differences in gravitational forces, to different forces generated by tidal forces of the Sun and Moon ... The relative importance of each of these factors and their relationship to each other is unclear.

The lithosphere with separate and distinct tectonic plates moves on the fluid-like (viscous-elastic solid) asthenosphere. Plate motions range up to a typical $10-40 \mathrm{~mm} /$ year to about $160 \mathrm{~mm} /$ year. The location where two plates meet is called a plate boundary; these boundaries are associated with earthquakes and the creation of topographic features such as mountains.

The energy required to move the tectonic plates causing earth quakes can be traced to gravitational waves. There are three Earth Quakes on $16^{\text {th }}$ April 2016 with Epicenters Falling on a Circle close to March Equinox. These three earthquakes occurred:

1. Ecuador earthquake (Ecuador lies above the destructive plate boundary) at 06:58 PM

2. Afghanistan Pakistan Border (Himalayas) at 02:10 AM, see Fig. 1a, and

3. Kumamoto Japan (North American, Pacific, Eurasian and Philippine plates come together here) a series of earthquakes since April 14, the main one at 01:25 AM on $16^{\text {th }}$ April. There must have been gravitational waves zipping across the earth in this period causing these earthquakes, unfortunately this is not recorded to make it conclusive.

The epicenter of the earth quake at a depth of $210.4 \mathrm{~km}$ in the gorge of bottom river of Afghanistan-Pakistan border is pictured in Fig. 1b with a cross-section at AA.

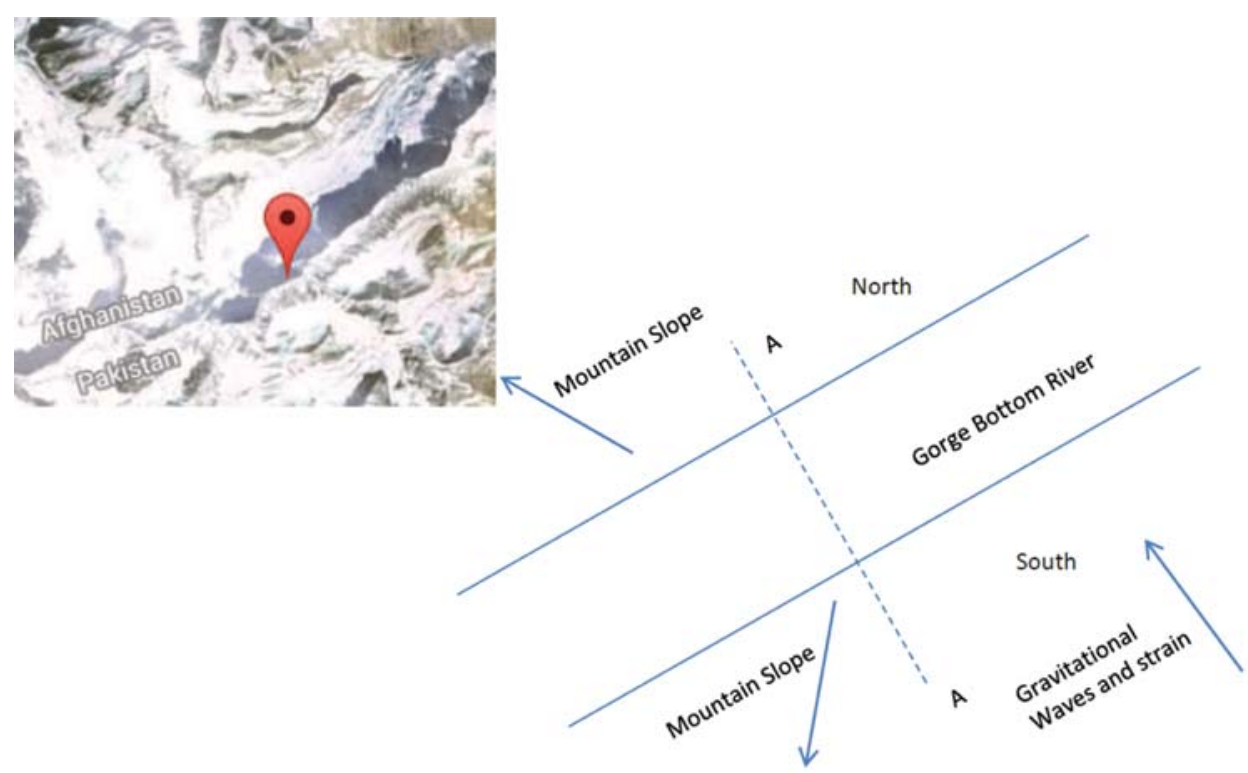

Fig. 1a Afghanistan Pakistan Border (Himalayas) Earthquake at 02:10 AM on $16^{\text {th }}$ April 2016

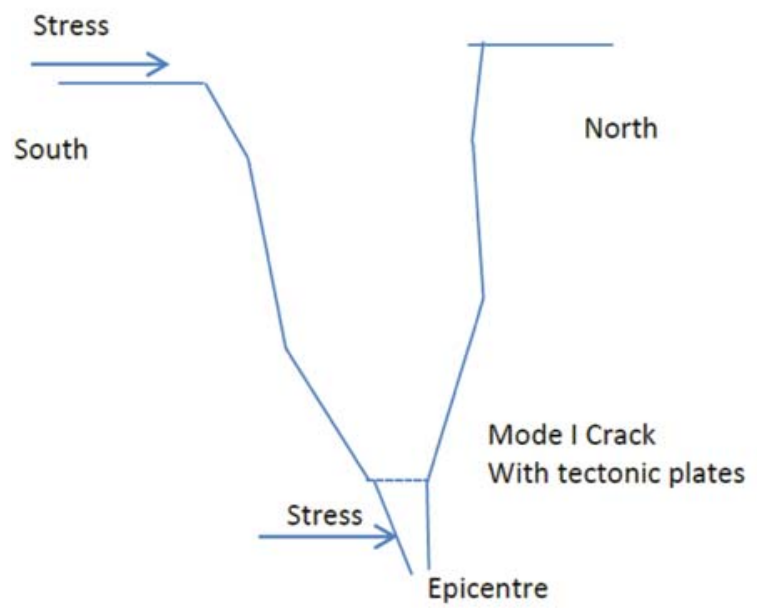

Fig. 1b The Epicenter of Earthquake in Fig. 1a 


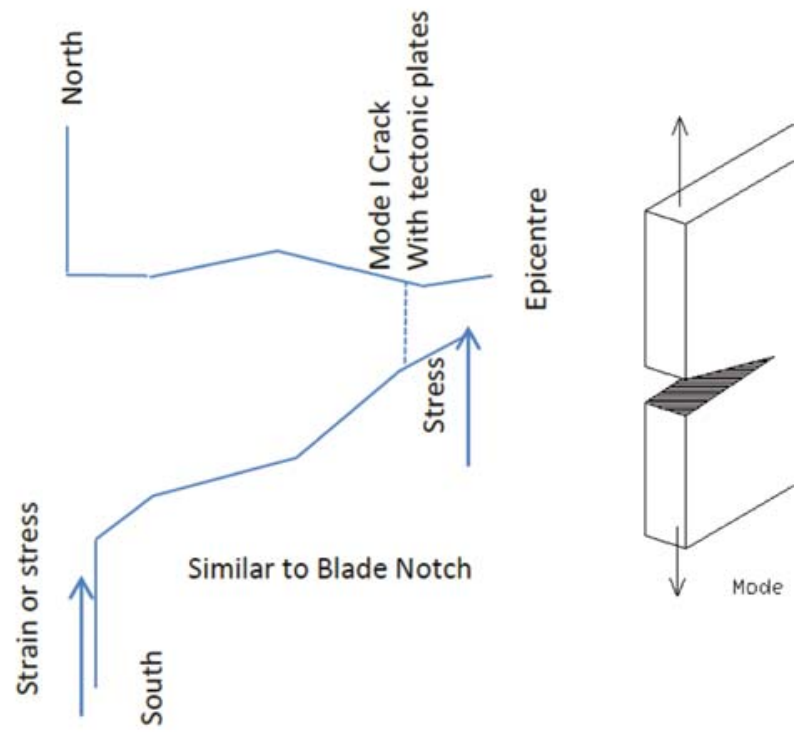

Fig. 1c Fatigue at Epicenter and Earthquake on April $16^{\text {th }} 2016$ in Himalayas

The same picture is put side by side with Mode I crack; see Fig. 1c which frequently occurs in all structures causing a fatigue failure. At the epicenter the stress concentration factor can be almost infinity causing a Himalayan Overlapping Thrust Fault to open up and create an earthquake.

While this source of energy is expected to be continuous, it is too small for the benefit of mankind and therefore cannot be a sustainable energy to the Earth. Stephen Hawking (2017) believes that life on Earth is at an ever-increasing risk of being wiped out by a disaster, such as asteroid strikes, epidemics, over-population, sudden nuclear war, a genetically engineered virus, or other dangers including climate change. The risk apart, the mankind would like to have sustainable abundant energy; what could that be? Bring Sun directly to the Earth.

It may be noted that there have been four instances of gravitational waves measurement and at all these times earthquakes have been reported, see https://earthquake-report.com, giving rise to speculation that it is the energy from gravitational waves that is responsible to generate the earthquakes.

\section{Sustainable Energy - Sun's Fusion on the Earth}

Modern atomic theory can be attributed to Rutherford (1911). Heavier atoms can be split to have atomic fission and lighter atoms can be fused to have fusion and release energy from the matter. It's the fusion that is most attractive as sustainable energy by bringing the fusion of light atoms and isotopes as it happens in the stars of this Universe. Eddington (1920, 1926) first proposed the internal constitution of the stars as fusion that occurs in the Universe.

Fusion in nuclear physics is the joining of two atomic nuclei. It occurs in stars all over the universe, including our Sun, and is what provides our energy. For fusion to work, extremely high energies are needed to fuse the nuclei together to overcome the electrical repulsion (also known as the Coulomb barrier) between two positively charged nuclei, so that they get close enough to have the strong nuclear force bind the nuclei. This nuclear force has an effective range of around 10-15 meters, which is why fusion occurs most easily in stars, where a high density and temperature environment exists. The density and temperature are the primary factors in determining the probability of the nucleons fusing in the star.

Most of the energy generated within the Sun is created from a sequence of reactions that burns hydrogen into helium, known as the protonproton reaction. In our Sun, these reactions occur in the central region, where the density is increased to 100 times that of water, sending temperatures to about 15 million $\mathrm{K}$ where the hydrogen is ionized, or stripped of their electrons, creating plasma. The heat provides enough energy for the hydrogen ions to collide with enough force to overcome the repulsion between these positively charged nuclei and fuse them. One of the resulting two protons that just fused decays into a neutron through $ß+$ decay, forming a deuteron. The decay process also releases an antielectron, or positron, and a neutrino. The positron will later collide with an electron and annihilate each other, releasing two gamma rays, which are high-energy photons. The neutrino interacts very weakly with matter, and will pass right out of the sun. The newly formed deuteron $\left({ }^{2} \mathrm{H}\right)$ may collide into another hydrogen nucleus, creating the helium isotope ${ }^{3} \mathrm{He}$ and causing the release of a gamma ray. When two of these ${ }^{3} \mathrm{He}$ isotopes collide, two of its protons are released, creating ${ }^{4} \mathrm{He}$. This sequence of nuclear reactions, known as the proton-proton reaction, can be written as:

$$
\begin{aligned}
& \text { 1. }{ }^{1} \mathrm{H}+{ }^{1} \mathrm{H} \cong{ }^{2} \mathrm{H}+\text { positron }\left(B^{+}\right)+\text {neutrino } \\
& \text { 2. }{ }^{2} \mathrm{H}+{ }^{1} \mathrm{H} \cong{ }^{3} \mathrm{He}+\text { gamma ray } \\
& \text { 3. }{ }^{3} \mathrm{He}+{ }^{3} \mathrm{He} \cong{ }^{4} \mathrm{He}+{ }^{1} \mathrm{H}+{ }^{1} \mathrm{H}
\end{aligned}
$$

Steps 1 and 2 must be done twice for each step $3 .{ }^{3} \mathrm{He}$ and ${ }^{4} \mathrm{He}$ are stable isotopes. ${ }^{4} \mathrm{He}$ needs even higher energies to fuse, since the repulsion between the two pairs of positively charged protons in helium is even greater than the repulsion between only two hydrogen nuclei.

To cause fusion here on Earth, the atoms to be fused must be in the form of plasma. To achieve this new state of matter, a gas is heated, causing the atoms to move very rapidly. At a high enough temperature, the electrons become separated from the nuclei, thus creating a cloud of charged particles, or ions. Sun, Stars, Lightning, and the gas in neon signs are all plasmas. Even higher temperatures are needed to cause the nuclei to collide and fuse. Such a condition where the thermal energy (how hot it is) of nuclei is high enough to fuse despite their repulsion is called thermonuclear.

1. Temperature. Simply put, the hotter the plasma, the more fusion occurs. This is because the nuclei will have enough energy to overcome their electromagnetic repulsion and fuse. Actually, it is possible to go too fast, causing the nuclei to zoom by each other, not staying together long enough to fuse. For the Deuterium-Tritium fusion reaction to work, the temperature must reach around at least 100 million $\mathrm{K}$.

2. Density. The more dense the plasma, the higher the probability of collision.

3. Containment. The plasma must also be confined in order for fusion to take place. No material can contain this hot plasma; it would either damage the material or the material would cool the plasma down, so an alternative is needed. Sun uses its huge gravitational force to squeeze the particles together. However, here on Earth, using gravity is not feasible. Instead, this confinement process is achieved through one of two methods: magnetic confinement or inertial confinement.

4. Confinement Time. The longer the energy confinement time, the more likely the plasma will sustain the high temperature and cause the fusion reactions to become self-sustaining. This confinement time is simply how long it takes the energy of confined plasma to leak out. 
Igor Tamm and Andrei Sakharov of Soviet Union in 1951 proposed a tokamak system for the realization of Controlled Thermonuclear Fusion on the basis of toroidal magnetic thermonuclear reactor.

It's in 1968 at the meeting held in Novosibirsk the Soviet delegation announced the first tokamak (toroidal chamber with a magnetic coils) design reaching performance levels at least an order of magnitude better than any other device. Within these machines, magnetic fields are used to contain the charged particles that compose the hot plasma and keep it away from the chamber walls. The charged particles, like those in plasma, will travel along the lines of a magnetic field. By arranging magnetic fields in just the right way, it was possible to trap the plasma within the fields.

While the plasma is held, it can be heated through a combination of microwaves, particle beams, and the heating generated from currents flowing through the plasma. The plasma density in a magnetically confined reactor is roughly $10^{15}$ particles $/ \mathrm{cm}^{3}$, which is thousands of times less dense than that of air at room temperature. In this design, the chamber is toroidal, or doughnut-shaped, thus having no open ends. The magnetic field is generated through the current running in the coils that are wrapped around the reactor. The field is stronger towards the center, causing the plasma to tend towards the outer wall. However, another magnetic field generated by a current going through the plasma itself combines with the coils' magnetic field to create magnetic lines that spiral around the torus. This spiraling counteracts the drifting effect on the plasma because of the strong inner field, and effectively traps the plasma. The cross-section of a tokamak is shown schematically in Fig. 2a

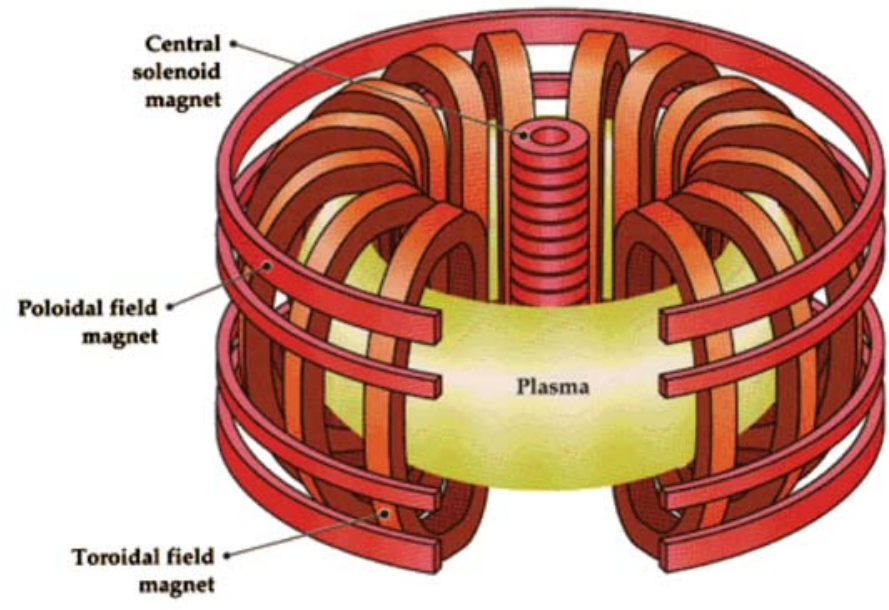

Fig. 2a Cross-section Sketch of a Tokamak

JET, the Joint European Torus, is the world's largest operational magnetic confinement plasma physics experiment, located at Culham Centre for Fusion Energy in Oxfordshire, UK based on a tokamak design. In 1997, JET set the current world record for fusion output at $16 \mathrm{MW}$ from an input of $24 \mathrm{MW}$ of heating. This is also the world record for factor $Q=16 / 24$ at 0.67 . A Q of 1 is breakeven. This is shown in Fig. $2 b$. It has a major radius of 3 meters, and the D-shaped vacuum chamber is 2.5 meters wide (the minor radius) and 4.2 meters high. The total plasma volume within it is 100 cubic meters, about 100 times larger than the largest machine in production when the JET design began, see Wesson (1999).

JET was one of the first tokamaks to be designed to use a D-shaped vacuum chamber. Electrical power was needed to supply the currents in the toroidal and poloidal field coils, a similar power being required for each. JET was designed to allow a pulse repetition rate of one every 15 minutes. Each pulse would call for a total power of up to 800MW - the output of a medium sized power station. In previous machines this pulsed load had been dealt with using heavy flywheels driven up to speed by motor generators, the energy in the flywheel then being extracted during the plasma pulse. The system used for JET combined this procedure with several hundred megawatts taken directly from the electricity grid.

Kamerlingh Onnes (1911) discovered superconductivity at very low temperatures. Since three magnet systems are required, the conventional electromagnets have to be supplied with energy, so much so a tokomak remained energy deficient; we have to supply energy for demonstration of fusion. Sharma (2015) discussed basics of design and application of superconducting magnets in accelerators and fusion reactors.
ITER is a joint international research and development project that aims to demonstrate the scientific and technical feasibility of $500 \mathrm{MW}$ fusion power. The partners in the project - the ITER Parties - are the European Union (represented by EURATOM), Japan, the People's Republic of China, India, the Republic of Korea, the Russian Federation and the USA. ITER is under construction in Europe, at Cadarache in the South of France. India joined the group on November 21 2006. Once this is successful, a prototype commercial reactor will be built, and if that works, fusion technology as a sustainable energy to this earth will be rolled out.

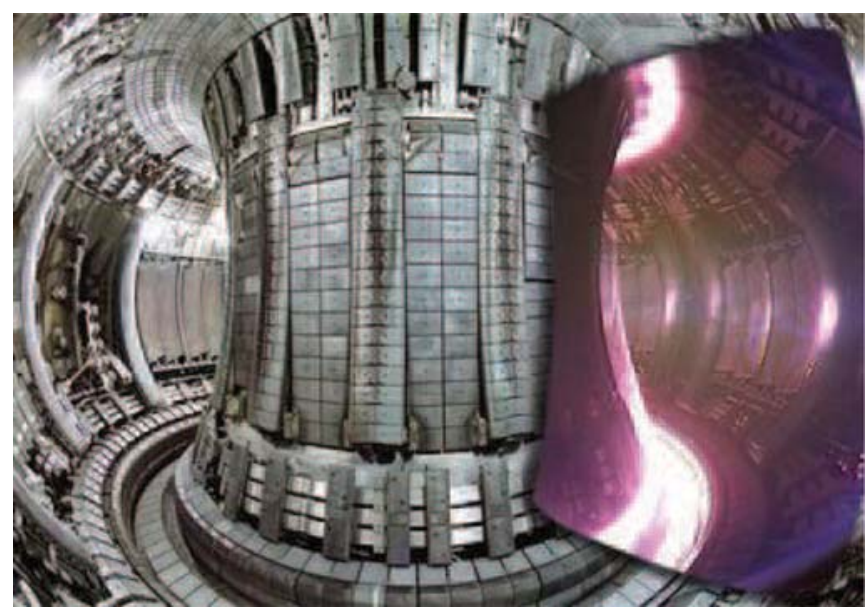

Fig. 2b JET tokamak superimposed with an image of a plasma

In fusion reactor designs, superconductors (which suffer no resistive power loss) are used to generate the magnetic fields that confine the 100 million ${ }^{\circ} \mathrm{C}$ plasma. While increasing magnetic field strength offers potential ways to improve reactor performance, conventional low-temperature superconductors suffer dramatic drops in current carrying ability at high magnetic fields. Now, the emergence of high-temperature superconductors that can also operate at high magnetic fields 30 Tesla or higher opens a new, lower-cost path to fusion energy.

For tokamak design, the field strength limits are primarily determined by the maximum allowable stresses in the structural components holding the magnet together, and not by the intrinsic limits of the superconductors. There are several technology challenges remain to be solved, however, the world-wide experience from tokamak experiments provide the support for power producing fusion reactors.

\section{ITER (International Thermonuclear Experimental Reactor)}

ITER in Cadarache, France was engaged in the design of $500 \mathrm{MW}$ fusion reactors, see Fig. 2c. The Cryostat is $24 \mathrm{~m}$ high and $24 \mathrm{~m}$ in dia weighing around 23350 tons. It has a central solenoid, toroidal and poloidal field coils forming the superconducting magnetic field for the plasma

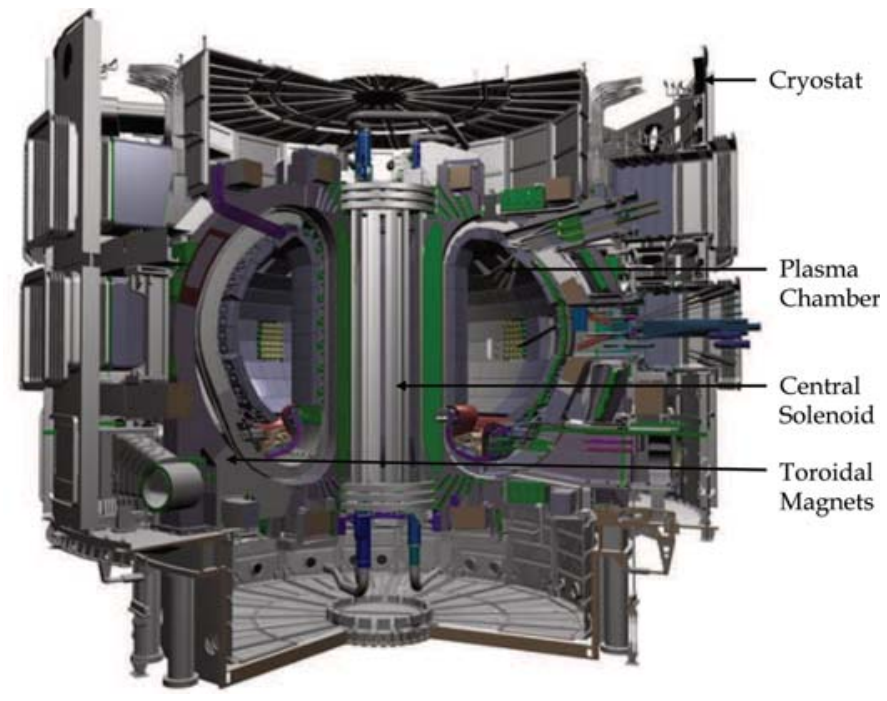

Fig. 2c ITER Cryostat 
contained in the tokomak maintained in a molecular level vacuum. The plasma of volume $840 \mathrm{~m}^{3}$ is at 100 million degrees centigrade temperature or more that moves in a toroidal path under the influence of the magnetic field. The vacuum vessel is made of 9 sectors comprising of a blanket with 440 modules. The cryostat is the most complex multi-physics system. Two isotopes of hydrogen, Deuterium and Tritium form the plasma under high pressure and temperature. Whereas Deuterium is a stable and available in sea water, Tritium is produced by the reactor itself.

Producing Tritium in the Power Plant is the most important aspect in design. Tritium is bred in the reactor in Tritium Breeding Modules (TBM). Fig. $3 a$ shows the location of TBM in the cryostat and Fig. $3 b$ shows a schematic of radial buildup of fusion breeding blanket.

Lead-Lithium Cooled Ceramic Breeder (LLCB) blanket module breeds Tritium and extracts heat from the fusion reactor. This module is heated by the $14 \mathrm{MeV}$ neutrons and the surface heat flux from the fusion Plasma and is kept in front of the Plasma. In the LLCB Tritium breeding module the coolant is Lead-Lithium (LL) flowing around the ceramic breeder zone. The Plasma facing surface of the module is the First Wall (FW) made of Ferrite Martensitic Steel (FMS) cooled separately by high pressure Helium gas. The flowing LL experiences very high magnetic field in the reactor environment $(\sim 4$ Tesla) in transverse direction and develops high MHD pressure drops due to induced current interaction with the magnetic field. This modifies the flow velocity profiles and turbulence characteristics, and hence the heat transfer and MHD pressure drop in the flow channels and manifolds. The engineering design of the LLCB Tritium breeding module should be an optimized design in various aspects such as, mechanical, thermal, structural, thermal hydraulics, thermo mechanics, magneto hydrodynamic pressure drops and electromagnetic loads. The TBM system includes several associated equipment, such as primary and secondary coolant circuits and components, Tritium management components, liquid breeder loop for liquid breeder TBM's, instrumentation packaging and control system, safety detection systems and valves.

Since it faces plasma (FW), TBM has to be cooled such that its walls, particularly facing Plasma can withstand this heat. He is circulated to provide the necessary cooling in TBMs. Lithium Titanate is used to breed Tritium in the breeders. Beryllium is used in Helium Cooled Solid Breeder (HCSB-TBM) to provide for extra neutrons. LLCBs are dual cooled (DCLL), $\mathrm{He}$ and $\mathrm{Pb}$-Li are used; the $\mathrm{Pb}$-Li providing additional neutrons. Figs. $3 b$ and $3 c$ give Schematics of radial buildup of fusion breeding blanket and concept of LLCB-TBM.

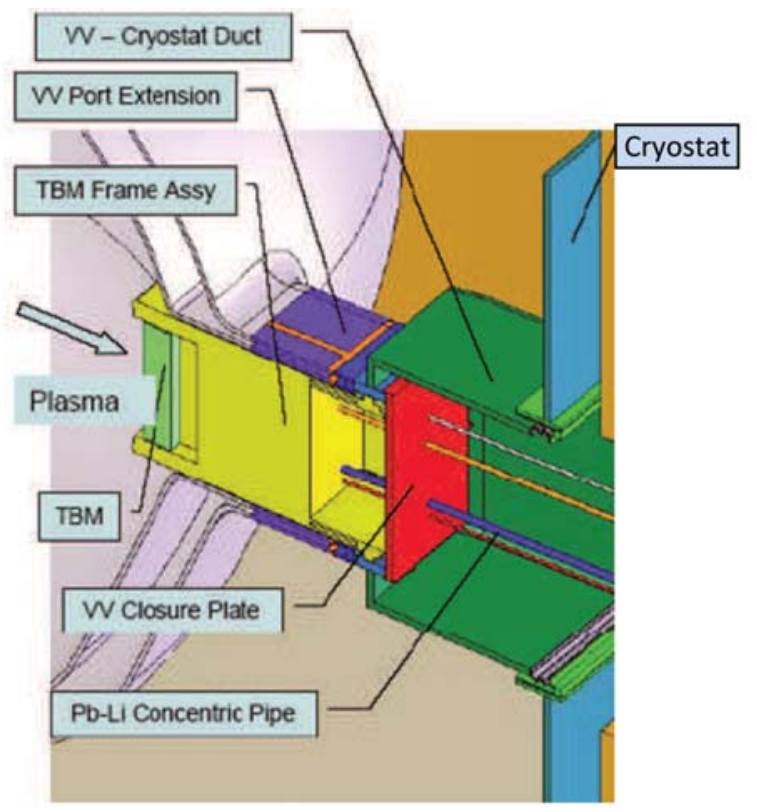

Fig. 3a Location of TBM in Cryostat

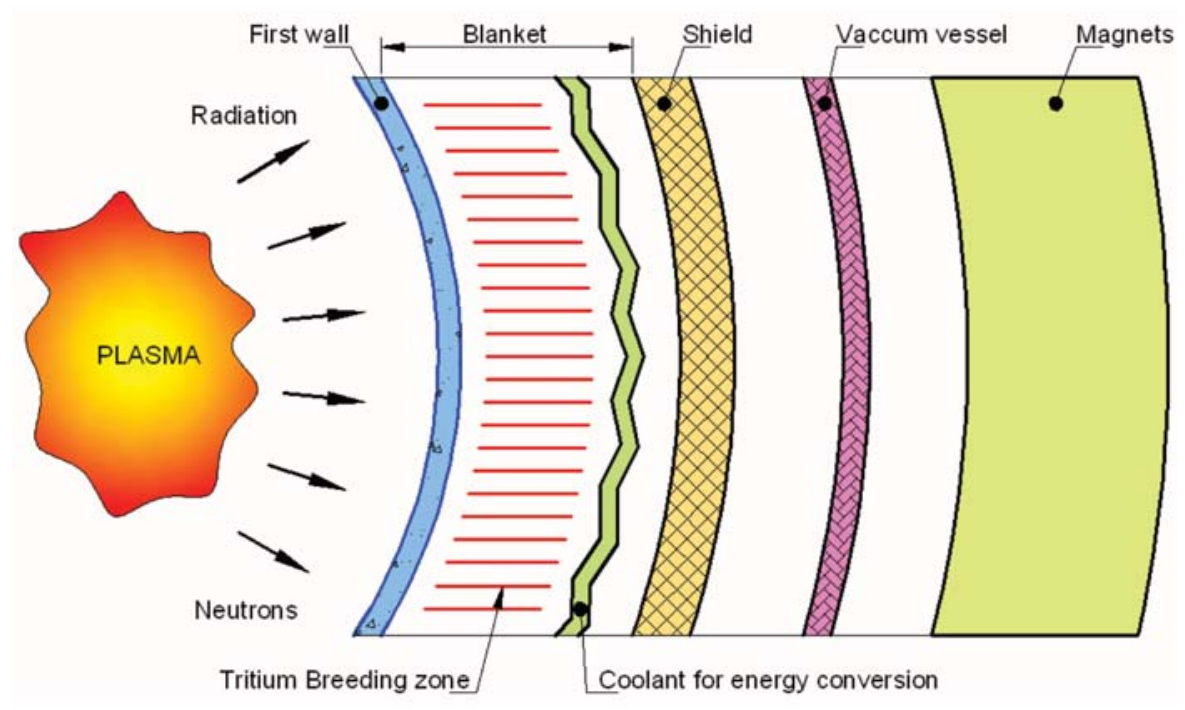

Fig. 3b Schematic of radial buildup of fusion breeding blanket 
The TBM assembly consists of FW, top (covering) plate, bottom (covering) plate, ceramic breeder zones, inlet and outlet channels for $\mathrm{Pb}$ Li flow, Helium purge lines, back plate, supports for TBM, piping and manifolds. The FW along with top plate, bottom plate and back plate form a closed enclosure. The TBM is inserted in a water-cooled steel frame, $20 \mathrm{~mm}$-thick, which provides a standardized interface with the ITER basic structure, including thermal insulation of the basic machine. TBM is mechanically connected to the back wall of the frame through flexible supports. It is supported at the back side with dowel pins and support keys. The ambient condition for TBM excluding the wall facing plasma is a vacuum of the order of $10^{-8}$ torr.

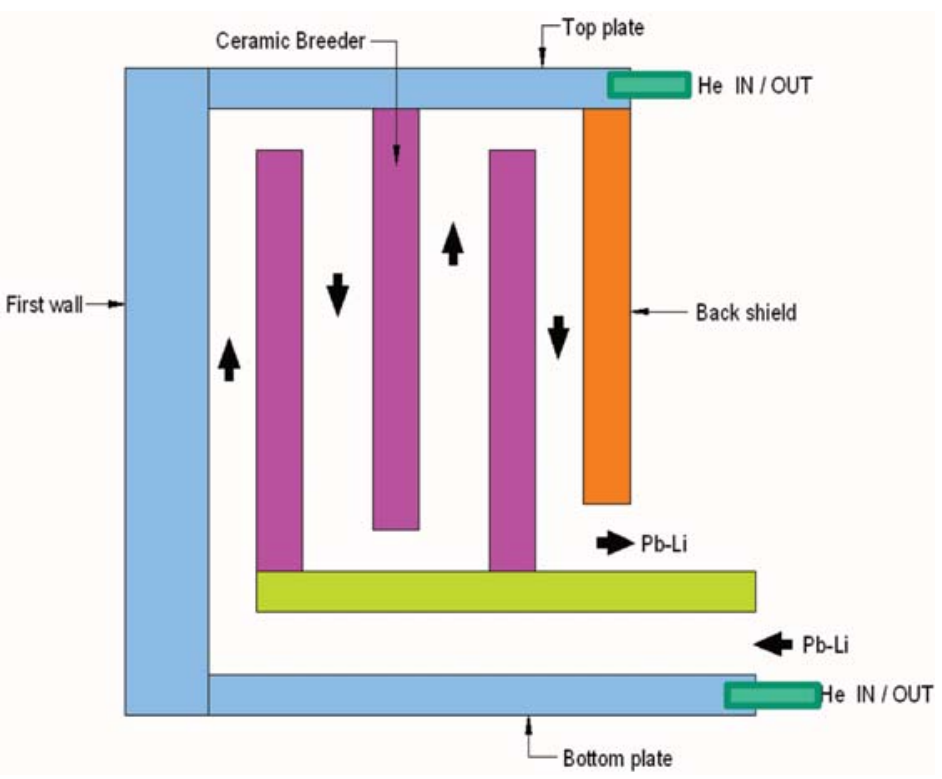

Fig. 3c Schematic of concept LLCB-TBM

Major functions of LLCB are: 1. Transmutation reaction between Lithium in blanket and neutrons from Plasma that generates Tritium and heat, 2. Lead acting as a neutron multiplier increases the number of neutrons and hence the higher Tritium breeding and 3. Heat extraction from the test blanket by Lead Lithium and Helium. Lead Lithium continuously circulates and acts as a coolant for the ceramic breeder, Helium acts as a coolant for the FW, top plate, bottom plate and back plate. The FW of TBM receives heat flux from Plasma. It has two Helium circuits (Purge Helium circuit and Coolant Helium circuit) and a Lead Lithium circuit. The Purge Helium circuit is for extracting Tritium and Coolant Helium circuit is for cooling FW, top and bottom plate and back plate. The Lead Lithium circuit acts as a coolant for ceramic breeder and neutron multiplier for higher Tritium breeding. Wong et al (2008) gave an overview of liquid metal TBM concepts and programs in progress in different countries as given in Table 1.

Table 1 Liquid TBMs developed in the various countries

\begin{tabular}{|l|l|l|}
\hline Country & Name of TBM & $\begin{array}{l}\text { Liquid } \\
\text { Metal } \\
\text { Used }\end{array}$ \\
\hline US & DCLL - Dual Coolant Lead Lithium & PbLi \\
\hline EU & HCLL - Helium Cooled Lithium Lead & PbLi \\
\hline Korea & HCML - Helium Cooled Molten Lithium & Li \\
\hline India & LLCB - Lead-Lithium Cooled Ceramic Breeder & PbLi \\
\hline China & DFLL - Dual Functional Lithium Lead & PbLi \\
\hline
\end{tabular}

A team of 18 engineers envisaged through discussions and workshops the design of LLCB to be 1. CFD analysis of the baseline Test Blanket Module (TBM) to find out the temperature, pressure and velocity distribution, 2. Optimize the flow path if required to meet the design criteria, 3. MHD analysis to estimate the pressure drop due to induced current interaction with magnetic field, 4. FEA analysis of flow optimized design, and 5. Optimize the TBM design to meet required structural design criteria.

The Dual Coolant, Pb-17Li (DCLL) TBM is designed using low activation Ferrite steel as structural material and $\mathrm{He}$ as a coolant for the first wall and structure. The TBM is designed to occupy one poloidal half section of the equatorial test port designated for testing blanket modules. It is mounted inside a water cooled frame designed to hold two different test modules.

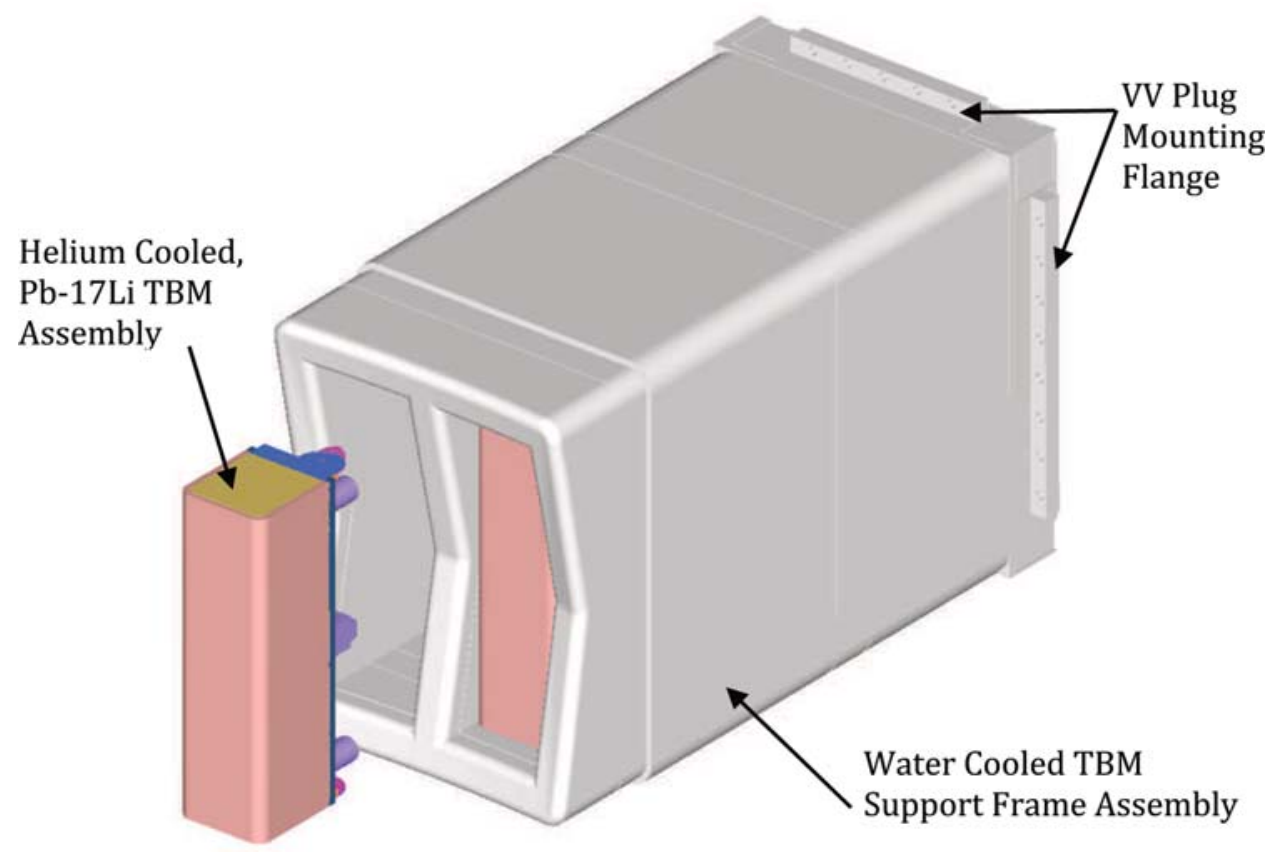

Fig. 4 LLCB TBM assembly installed in one of the half ports 
A baseline TBM model is first developed and used for CFD and thermo-mechanical analysis and optimization to fix flow paths, Electro Magnetic analysis for estimating EMag loads and structural design. Fig. 4 shows the TBM baseline model as it is inserted into the frame occupying the test port. The TBM is a rectangular structure with a faceted FW designed to match the surface of ITER shielding blanket. Its design provides flow channels for the $\mathrm{Pb}-17 \mathrm{Li}$ to flow poloidally at a slow speed while the He coolant is flowing throughout the TBM structure. The TBM assembly is shown in Fig. 5a.

The TBM is designed to use a center key support system to allow for supporting and centering it inside the frame. Peripheral (corner) support studs are to retain the TBM and stabilize it during operation, and allow for movement and expansion without restrain. The support key will be inserted into matching slot in the shielding block located behind the TBM, and the four positioning pins will be inserted into the shielding block and used to set the TBM into the proper position and provide the radial support needed at the top and bottom during operation as shown in Fig $5 b$.

As the TBM will be mounted inside water cooled support frame the overall TBM size is $1660 \mathrm{~mm}$ tall (Poloidal) by $480 \mathrm{~mm}$ wide (Toroidal) as shown in Fig. 5c. The total radial depth of TBM is $534 \mathrm{~m}$. The TBM is designed to use Helium as the primary coolant and $\mathrm{Pb}-17 \mathrm{Li}$ as the selfcooled breeding material. It is designed to accommodate the two flows internally and maintain a total separation between them. Also it is designed to withstand the maximum He pressures in case of an internal leak from the $\mathrm{He}$ into the $\mathrm{Pb}-17 \mathrm{Li}$ chambers. Fig. $5 \mathrm{~d}$ shows a cross-section of the $\mathrm{TBM}$ at the equatorial plane.

The FW assembly is designed to withstand the heat flux from the Plasma chamber and to maintain the TBM structure temperature below the allowable limits. It is a U shaped structure made of FMS material and designed with internal He coolant channel. The coolant channels are designed to allow multiple passes across the FW in order to maximize heat removal.

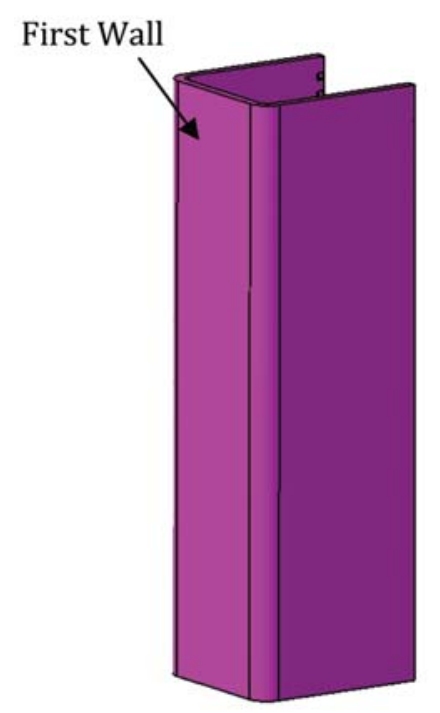

\section{Top plate}

\section{Assembly}

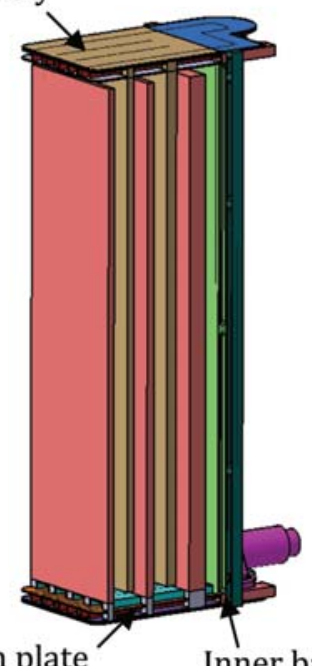

Inner back plate

Bottom plate

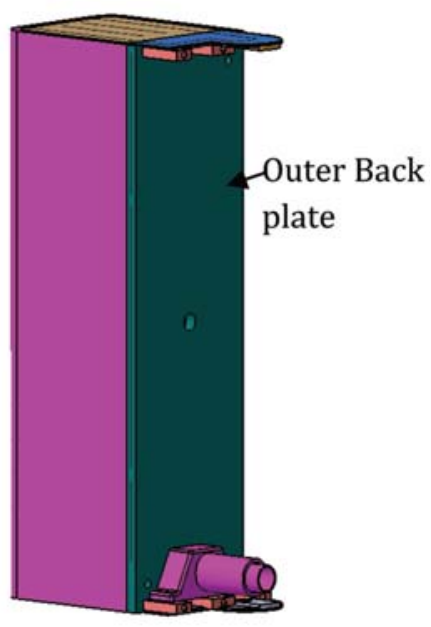

Fig. 5a LLCB TBM Assembly
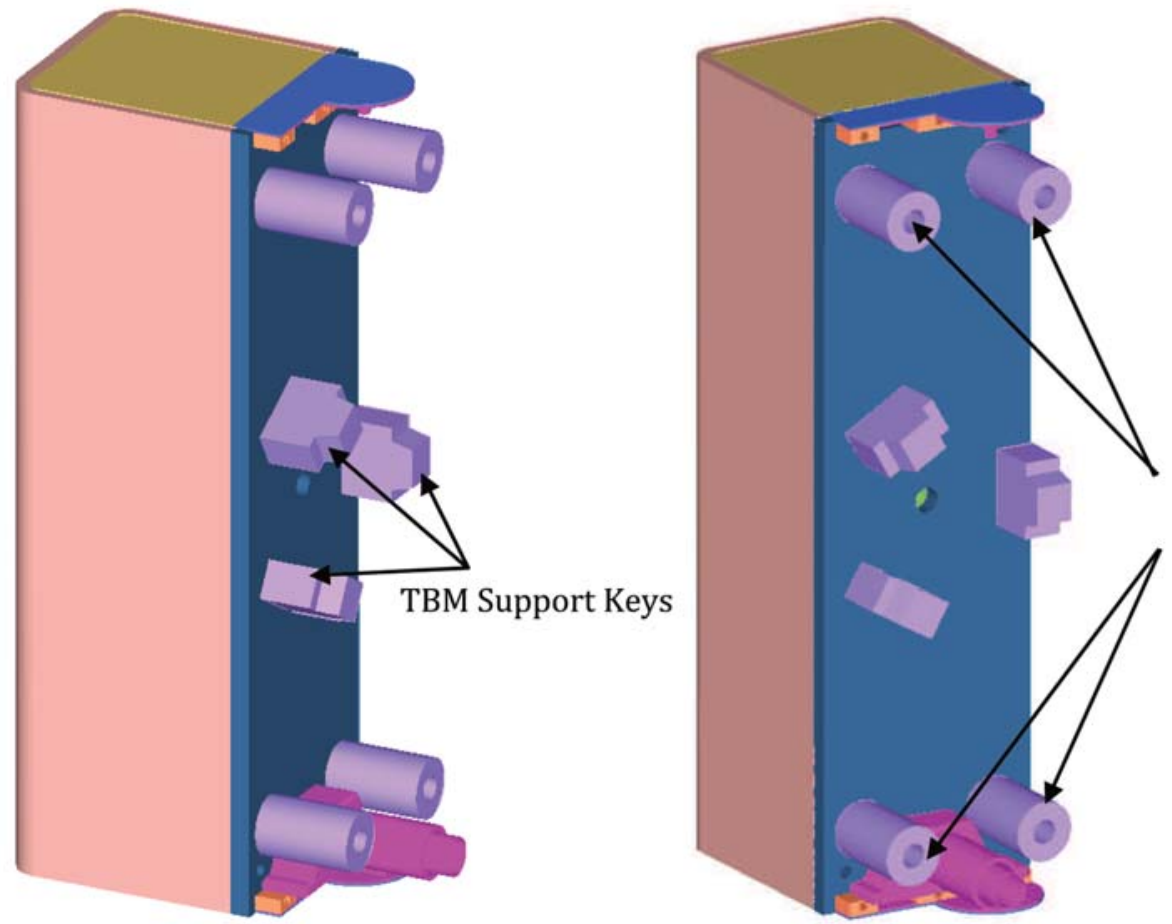

TBM Positioning and Support Keys

Fig. 5b TBM Support System 

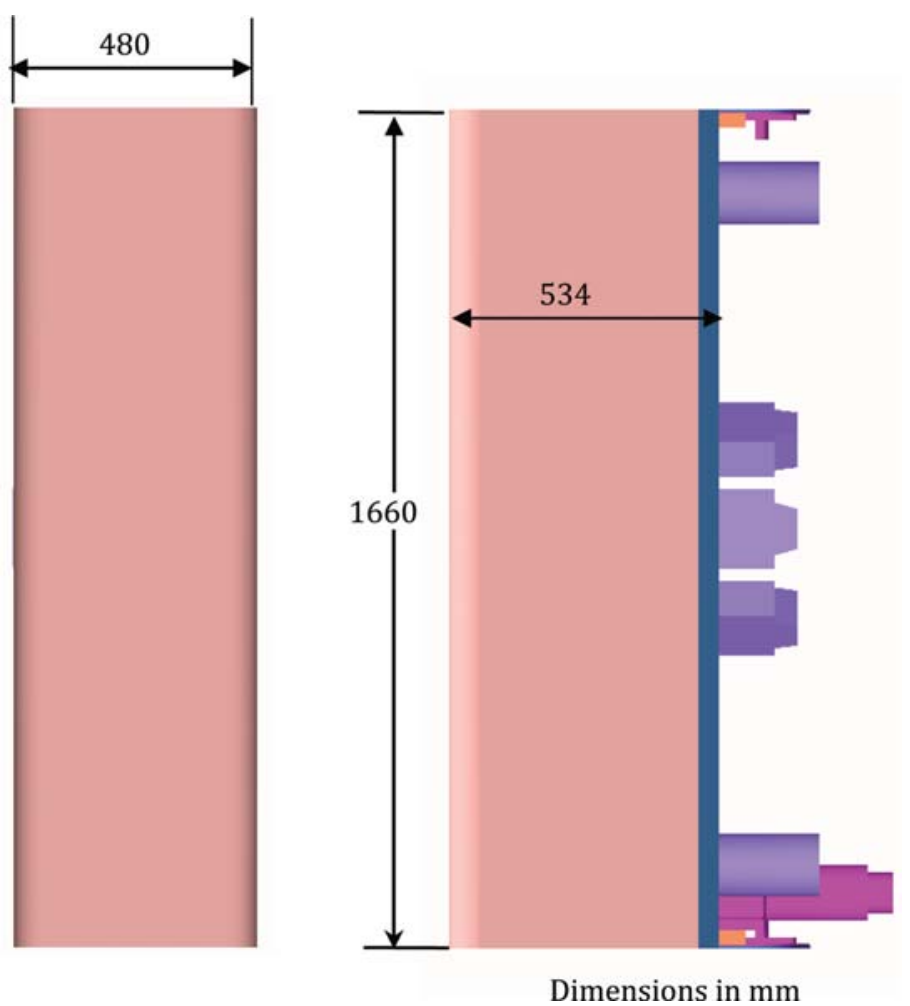

Fig. 5c LLCB TBM Dimensions

There are a total of 64 coolant channels, $20 \mathrm{~mm}$ wide by $20 \mathrm{~mm}$ depth in the TBM FW assembly. There are two circuits 1 and 2, both have 32 channels each and are interconnected by a series of manifolds in the back plates. The He flow between the two circuits is always in a counter flow arrangement to achieve a uniform temperature distribution across the FW surface. The two He circuits flowing through the FW channels are separated from each other and only mixed in the outlet manifold prior to entering into the outlet pipe. Circuit 1 of the He flow channels have openings at the edge face of the FW and these openings will match up with the outer back plate manifolds where the four passes are routed through. Circuit 2 on the other hand has the channel openings on the inner face of the FW. The FW structure is composed of a $4 \mathrm{~mm}$ thick FMS plate facing the plasma. This plate will have a $2 \mathrm{~mm}$ Beryllium layer on top of the FMS FW.

Complete design details including optimization are given by Rao et al (2008). Two exploded views of the LLCB are provided in Figs. 6a and

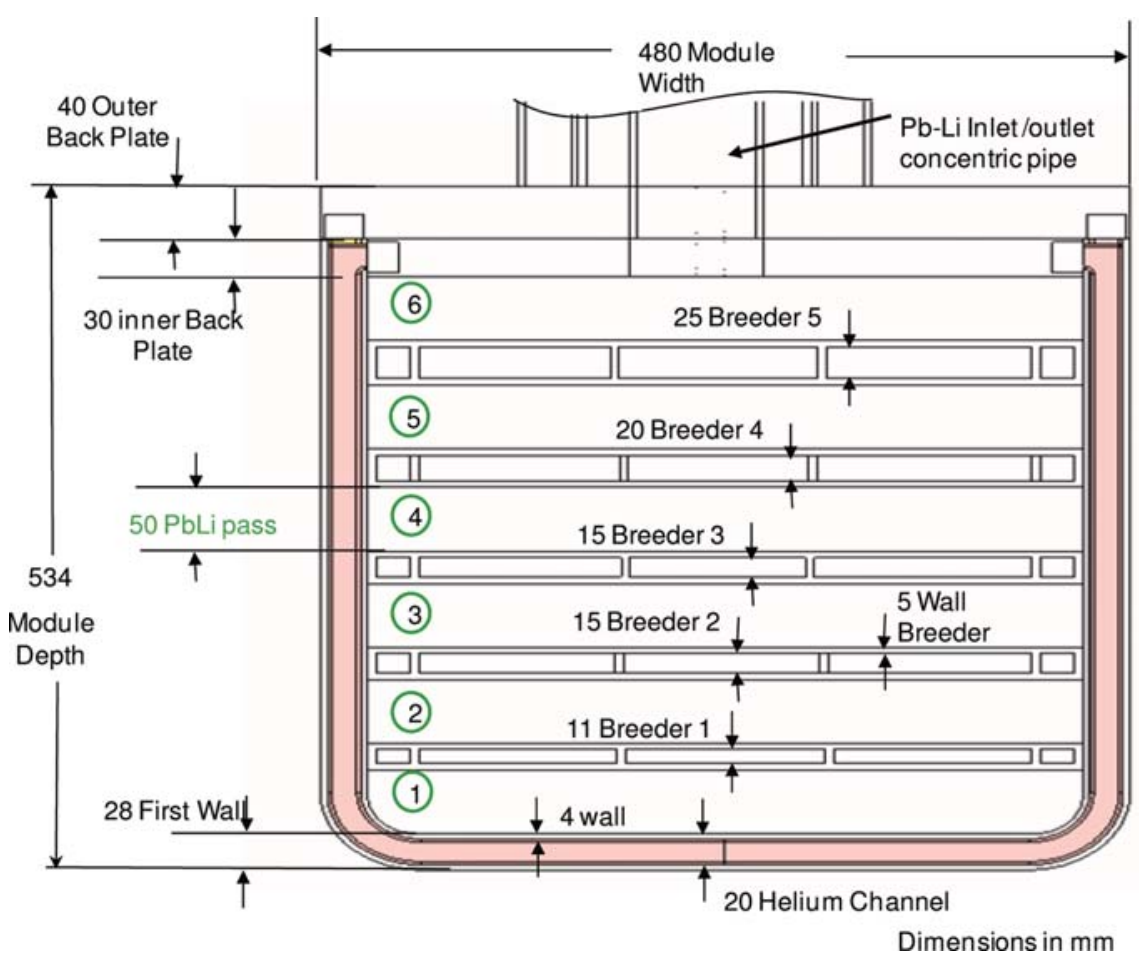

Fig. 5d Section view at TBM mid-plane without stiffeners 


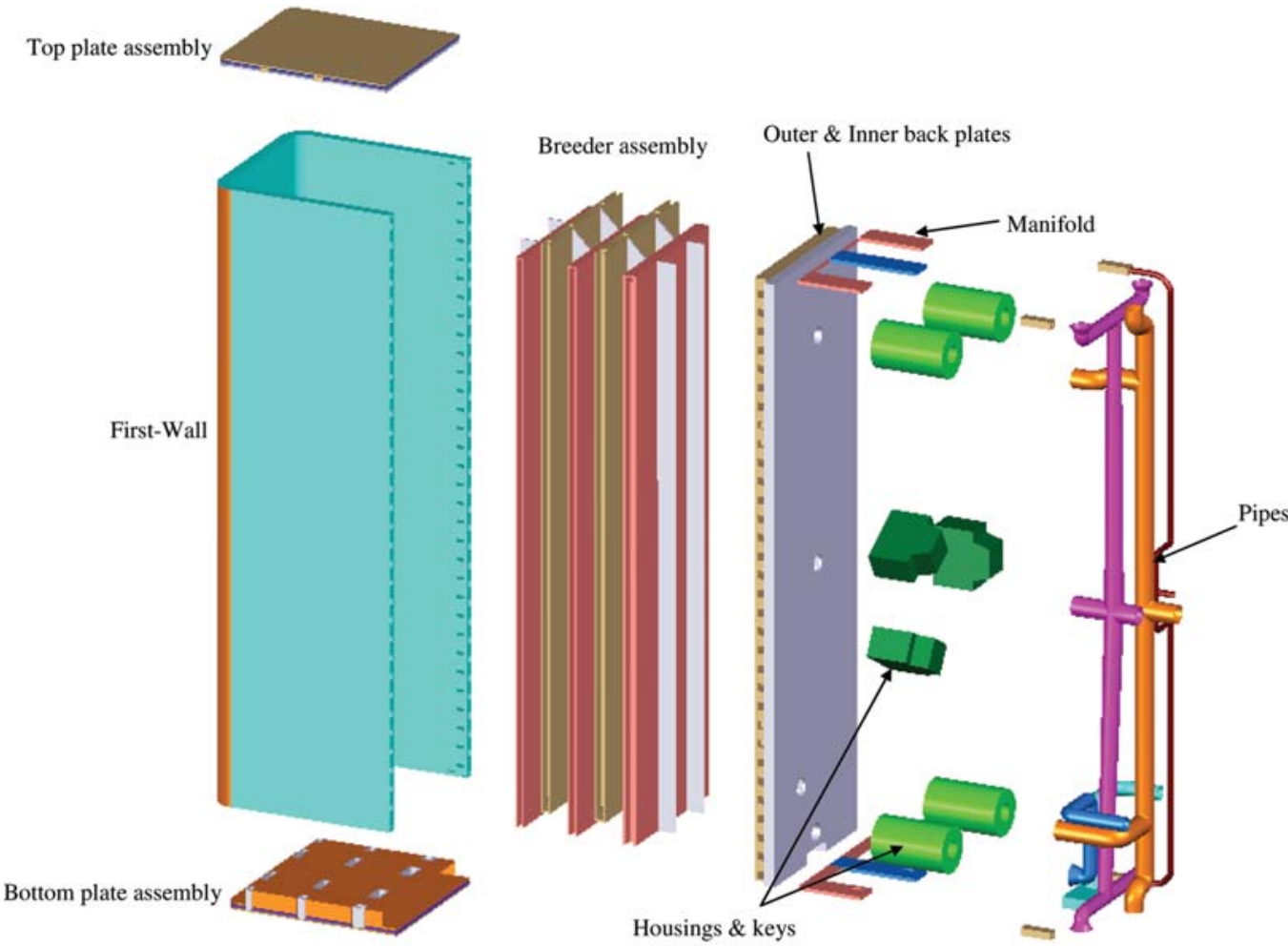

Fig. 6a Full LLCB exploded view

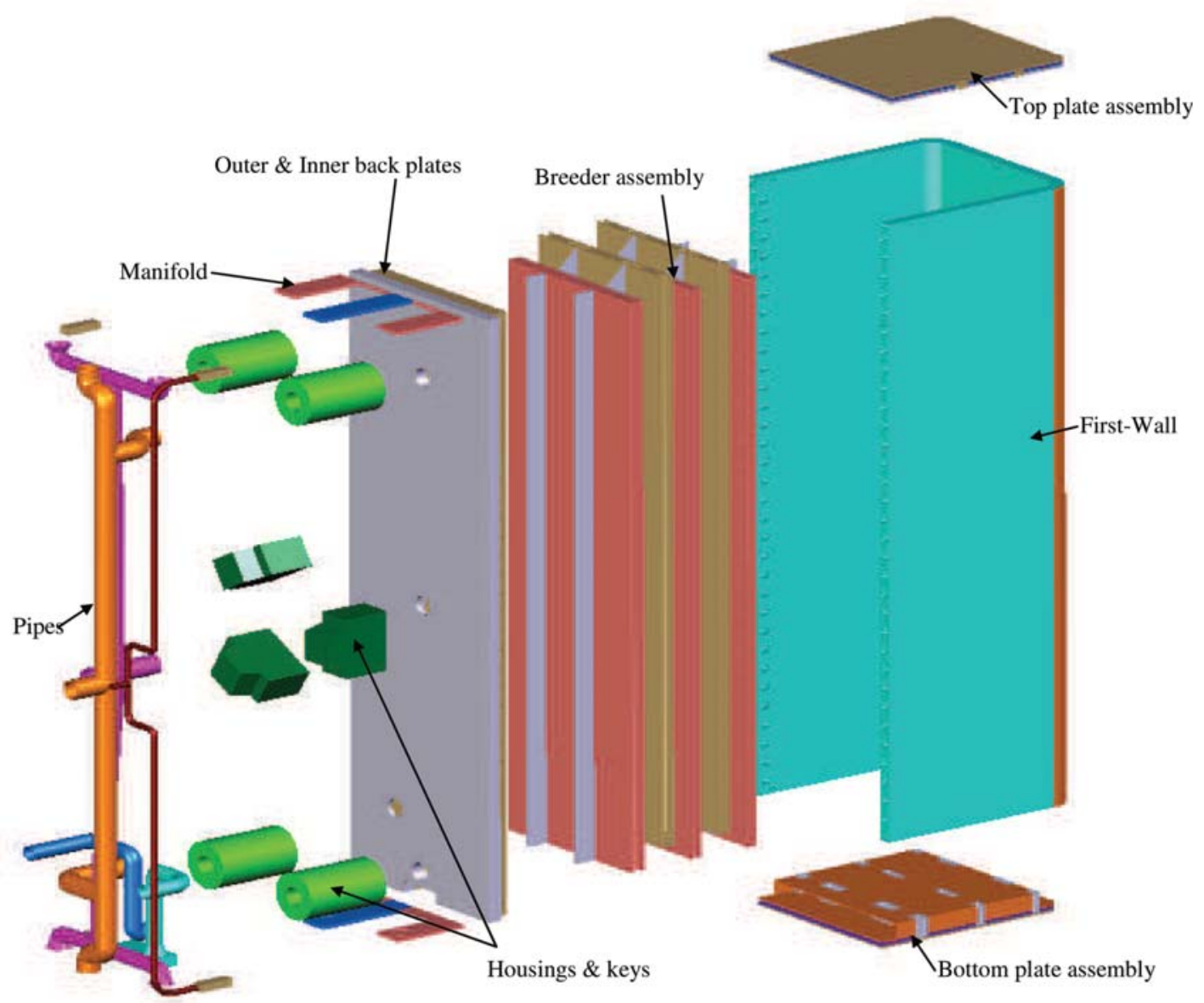

Fig. 6b Full LLCB exploded view 
$6 \mathrm{~b}$. This is the complete design of the LLCB designed entirely in India, a new technology allowing Sun to be brought on to this earth and provide pollution free energy.

\section{Current status of ITER}

Construction of the ITER Tokamak complex started in 2013 and the facility is expected to finish by 2021 . The plasma experiments are expected to begin in 2025 with full deuterium-tritium fusion experiments by 2035 . This will become the largest magnetic confinement plasma physics experiment in use with a plasma volume of 840 cubic meters. The first commercial demonstration fusion power station, named DEMO, is expected immediately after that. This will be truly a sustainable and abundant energy source for the world by bringing the Sun to the Earth.

\section{Conclusions}

There are only two forms of energy that the earth receives; 1. Gravitational Waves Energy and 2. Radiation from star Sun. The gravitational waves pass through earth at the speed of light almost continuously when two black holes merge. This energy received by earth is stored over millions of years is not sufficient for Man's needs. Radiation from Sun is the main energy source that can meet Man's needs partially as today; the energy stored in the form of Fossil fuels is not exhaustive and depleted rapidly. Other renewable forms from Sun are not sufficient to meet the requirements of mankind. The alternative left is to depend on energy conversion from matter as it happens in Sun and other stars in the Universe.

The atomic fission being not compatible for safety of mankind, fusion as it occurs in the stars seems to be the answer. Unlike fission that can be achieved with conventional technologies, fusion involves extreme technologies dealing with densities, pressures, temperatures, vacuum and superconducting magnets. This essentially means bringing Sun to the Earth and it has been demonstrated to be feasible. Future sustainable and abundant energy for mankind is to be rolled out from the initiatives taken by seven countries, ITER, during the next decade.

\section{References}

1. Chang Samuel Hsu, Paul R. Robinson, Editors, (2017), Springer Handbook of Petroleum Technology.

2. Cho, A., (2012). Higgs Boson Makes Its Debut After Decades-Long Search, Science. 337 (6091): 141-143.
3. Eddington, A. S., (1920), The internal constitution of the stars, Observatory, vol. 43, p. 341

4. Eddington, A. S., (1926), The internal constitution of the stars, Cambridge University Press.

5. Hawking, S., (2017) http://www.dailymail.co.uk/sciencetech/article-4468700/ Stephen-Hawking-says-leave-Earth-100-years.html

6. Hubble, E. (1929), A relation between distance and radial velocity among extragalactic nebulae. Proceedings of the National Academy of Sciences. Vol. 15 No. 3 , p. 168.

7. Michelson, A. A., (1881), The Relative Motion of the Earth and the Lumniferous Ether, American Journal of Science. Vol. 22, p. 120.

8. Onnes, K. H., Further experiments with liquid helium. C. On the change of electric resistance of pure metals at very low temperatures, etc. IV. The resistance of pure mercury at helium temperatures, Comm. Phys. Lab. Univ. Leiden; No. 120b, 1911.

9. Overbye, D., (2008) Let the Proton Smashing Begin, New York Times, July 29

10. Rao, J. S., (2011), History of Rotating Machinery Dynamics, Springer

11. Rao, J. S., (2016), Gravitational Waves and Engineering Aspects of Thermal and Earth Quakes of Earth, Proceedings of The Twelve International Conference on Vibration Engineering and Technology of Machinery, (VETOMAC-XII), Warsaw, Jablonna Palace, Poland, 7-9 (Wednesday - Friday) September, 2016

12. Rao, J. S., (2018) Creativity in Design - Science to Engineering Model, MAMT 3006, Mechanism and Machine Theory, 125, 52-79

13. Rao, J. S. et al. (2008). Design description document for the dual coolant Pb 17L (DCLL) test blanket module, Report to the ITER test blanket working group (TBWG), (2008), Institute of Plasma Research, India

14. Rao, J. S. \& Sankar, H. (2011a). Numerical Simulation of MHD Effects on Convective Heat Transfer Characteristics of Flow of Liquid Metal in Annular Tube. Journal of Fusion Engineering and Design, Vol.86, No.2-3, (March 2011), pp. 183-191.

15. Rao, J. S. and Hari Sankar, (2011b) Magneto Hydro-Dynamics and Heat Transfer in Liquid Metal Flows, chapter 4 in "Heat Transfer" Intech Publishers

16. Reitze, D., (2016) https://www.theguardian.com/science/video/2016/feb/11/wedid-it-scientists-announce-discovery-of-gravitational-waves-video

17. Rutherford, E., (1911), The Scattering of á and â Particles by Matter and the Structure of the Atom, Philosophical Magazine. Series 6, vol. 21, p. 669

18. Sharma, R.G., (2015) Superconductivity - Basics and Applications to Magnets, Springer

19. Wegener, Alfred (1912), Die Herausbildung der Grossformen der Erdrinde (Kontinente und Ozeane), auf geophysikalischer Grundlage, Presented at the annual meeting of the German Geological Society, Frankfurt am Main.

20. Wesson, J., (1999). The Science of JET, JET Joint Undertaking, Abingdon, Oxon, UK”. JET-R (99)13.

21. Wong, C. P. C. et al., (2008) Overview of liquid metal TBM concepts and programs, Fusion Engineering and Design, v. 83, p. 850 